\title{
Measuring Dynamic Structural Changes of Nanoparticles at the Atomic Scale Using Scanning Transmission Electron Microscopy
}

\author{
Annelies De wael@ ${ }^{1,2}$ Annick De Backer@, ${ }^{1,2}$ Lewys Jones $\odot,{ }^{3,4,5}$ Aakash Varambhia ${ }^{3, \dagger}$ \\ Peter D. Nellist $\odot,{ }^{3}$ and Sandra Van Aert $\odot^{1,2, *}$ \\ ${ }^{1}$ EMAT, University of Antwerp, Groenenborgerlaan 171, 2020 Antwerp, Belgium \\ ${ }^{2}$ NANOlab Center of Excellence, University of Antwerp, Groenenborgerlaan 171, 2020 Antwerp, Belgium \\ ${ }^{3}$ Department of Materials, University of Oxford, Parks Road, OX1 3PH Oxford, United Kingdom \\ ${ }^{4}$ Advanced Microscopy Laboratory, Centre for Research on Adaptive Nanostructures and Nanodevices (CRANN), \\ Dublin 2, Ireland \\ ${ }^{5}$ School of Physics, Trinity College Dublin, The University of Dublin, Dublin 2, Ireland
}

(Received 17 October 2019; accepted 30 January 2020; published 13 March 2020)

\begin{abstract}
We propose a new method to measure atomic scale dynamics of nanoparticles from experimental high-resolution annular dark field scanning transmission electron microscopy images. By using the socalled hidden Markov model, which explicitly models the possibility of structural changes, the number of atoms in each atomic column can be quantified over time. This newly proposed method outperforms the current atom-counting procedure and enables the determination of the probabilities and cross sections for surface diffusion. This method is therefore of great importance for revealing and quantifying the atomic structure when it evolves over time via adatom dynamics, surface diffusion, beam effects, or during in situ experiments.
\end{abstract}

DOI: 10.1103/PhysRevLett.124.106105

In the field of atomic resolution electron microscopy, quantitative methods are becoming increasingly important for a reliable structure determination of a nanomaterial in three dimensions [1-6]. However, such quantitative analysis tools largely focus on a stationary structure as a result of which the insight into the dynamics is lacking. Since the atomic structure of a nanomaterial can evolve over time via adatom dynamics $[7,8]$, surface diffusion and reconstruction [9-13], beam effects [14-17], or during in situ experiments $[18,19]$, a quantitative analysis at a single time-point is often insufficient to understand the atomic structure-properties relationship. Different time-resolving techniques, such as dynamic transmission electron microscopy [20,21], ultrafast electron diffraction [22-27], and ultrafast x-ray imaging $[28,29]$ have been developed in order to study structural dynamics with very high temporal resolution. Since these techniques sacrifice direct atomic resolution imaging, highresolution transmission electron microscopy [30], off-axis electron holography [31], or annular dark field (ADF) scanning transmission electron microscopy (STEM) $[32,33]$ are needed to obtain local structural information at atomic resolution and subsecond temporal resolution. This time resolution is adequate in order to study transient atomic scale phenomena [19,34-39]. The concomitant advantage for ADF STEM images is that the intensities are peaked at the atomic column positions and depend monotonically on the atomic mass number $Z$ and the thickness of the material enabling us to count the number of atoms in each atomic column. In this Letter, we propose a method for reliable atom counting from a sequence of ADF STEM images allowing us to measure the dynamic structural changes of nanoparticles.

From the ADF STEM images, we can quantify the total intensity of electrons scattered towards the annular detector from each atomic column. These are the so-called scattering cross sections [40,41], and are a suitable measure for reliable atom counting [42]. In pure-element nanomaterials, the number of atoms in each atomic column can be determined using these scattering cross sections [32,33,43-46]. When the atom-counting results are combined with a structural energy minimization [47-49], we can obtain a visualization of the 3D atomic structure from a 2D image without the need for the large electron doses and long acquisition times generally required for electron tomography. In order to quantify atomic scale dynamics, we will use a so-called hidden Markov model. Hidden Markov models were successful in other fields of science for applications such as speech recognition, sequence alignment of protein structures, electrocardiogram characterization, and conditionbased maintenance of industrial machines [50-54] and have optimal properties for modeling and analyzing time series data. Here, for the first time, we apply hidden Markov models to ADF STEM data.

A hidden Markov model consists of two layers: a "hidden" Markov chain state sequence and an observed sequence. In order to use hidden Markov models for atom counting, we model the number of atoms in each atomic column of the nanoparticle as the hidden states and the scattering cross sections estimated from the ADF STEM 
images as the observations. Table I in the Supplemental Material [55] summarizes the notation used throughout this Letter. The hidden state sequence is represented by a stochastic tensor $\boldsymbol{H}=\left(\boldsymbol{h}_{1}, \ldots, \boldsymbol{h}_{T}\right)^{\top}$, which contains the states $\boldsymbol{h}_{t}$ at each time $t$, with $T$ the total number of frames in the time series. In our method, the state $\boldsymbol{h}_{t}$ is a binary matrix with elements $h_{t g}^{(n)}=1$, if and only if at time $t$, the $n$th atomic column of the nanoparticle contains $g$ atoms. As such, we consider the number of atoms in each atomic column as a separate hidden state, and we can use the socalled factorial hidden Markov model [56], where the states and model probabilities are factorized over the atomic columns. The observed sequence is represented by the matrix $\boldsymbol{O}=\left(\boldsymbol{o}_{1}, \ldots, \boldsymbol{o}_{T}\right)^{\top}$, where $\boldsymbol{o}_{t}$ is the observed vector at time $t$ in the time series, with elements $o_{t}^{(n)}$, the scattering cross section of the $n$th atomic column at time $t$. When $T=1$, this approach is identical to the existing procedure for atom counting $[32,33,45,46]$. The (factorial) hidden Markov model is described by the joint probability density function of the hidden state sequence $\boldsymbol{H}$ and the observed sequence $\boldsymbol{O}$ :

$$
\begin{aligned}
& p(\boldsymbol{H}, \boldsymbol{O} ; \boldsymbol{\Omega}) \\
& \quad=p\left(\boldsymbol{h}_{1} ; \boldsymbol{I}\right) \prod_{t=2}^{T} p\left(\boldsymbol{h}_{t} \mid \boldsymbol{h}_{t-1} ; \boldsymbol{A}\right) \prod_{t^{\prime}=1}^{T} p\left(\boldsymbol{o}_{t^{\prime}} \mid \boldsymbol{h}_{t^{\prime}} ; \boldsymbol{\mu}, \sigma\right),
\end{aligned}
$$

with $\boldsymbol{\Omega}=\{\boldsymbol{I}, \boldsymbol{A}, \boldsymbol{\mu}, \sigma\}$ the parameters of the hidden Markov model. The joint probability density function of Eq. (1) consists of three contributions, schematically shown in Fig. 1. First, the initial probability distribution, defined as follows:

$$
p\left(\boldsymbol{h}_{1} ; \boldsymbol{I}\right)=\prod_{n=1}^{N} \prod_{g=0}^{G} l_{g}^{h_{1 g}^{(n)}},
$$

with $\boldsymbol{I}$ the vector containing all initial probabilities $l_{g}$ for an atomic column to have $g$ atoms in frame $1, G$ the maximum number of atoms in an atomic column, and $N$ the number of atomic columns. Once the state sequence is initialized, the transition probability describes how the states can change from frame to frame in the time series:

$$
p\left(\boldsymbol{h}_{t} \mid \boldsymbol{h}_{t-1} ; \boldsymbol{A}\right)=\prod_{n=1}^{N} \prod_{g_{1}=0}^{G} \prod_{g_{2}=0}^{G} A_{g_{1} g_{2}}^{h_{t-g_{1}}^{(n)}} h_{t, g_{2}}^{(n)} .
$$

This is the probability of state $\boldsymbol{h}_{t}$, given the previous state $\boldsymbol{h}_{t-1}$. The transition probabilities are summarized by the transition matrix $\boldsymbol{A}$, with elements $A_{g_{1} g_{2}}$ the probability that the number of atoms in an atomic column changes from $g_{1}$ in one frame to $g_{2}$ in the next frame of the time series. The Markov property imposes that the state sequence has no memory: the number of atoms in an atomic column in a frame only depends on the number of atoms in that atomic column in the previous frame, and does not depend on any earlier frames. The hidden Markov model does not impose further restrictions on the physical mechanism causing the changes in the atomic structure. Finally, for each frame, there is an emission probability that describes the probability of an observation, given the hidden state at that time. Ideally, all atomic columns with the same number of atoms result in the same scattering cross section. However, there are fluctuations due to a combination of different effects such as electron counting statistics, instabilities of the microscope, different vertical onset of columns of the same number of atoms, vacancies, relaxation at the boundaries, contamination, intensity transfer between columns, and the influence of neighboring columns of different number of atoms. Therefore, the scattering cross sections are regarded as a statistical draw from a Gaussian distribution, which defines the emission probability:

$$
p\left(\boldsymbol{o}_{t} \mid \boldsymbol{h}_{t} ; \boldsymbol{\mu}, \sigma\right)=\prod_{n=1}^{N} \prod_{g=0}^{G} \mathcal{N}\left(o_{t}^{(n)} \mid \mu_{g}, \sigma\right)^{h_{t g}^{(n)}},
$$

with $\boldsymbol{\mu}$ the vector containing the average scattering cross sections $\mu_{g}$ for an atomic column with $g$ atoms, and $\sigma$ the width of the Gaussian distribution, analogous to the approach followed in Refs. [33,45]. Furthermore, prior (a)

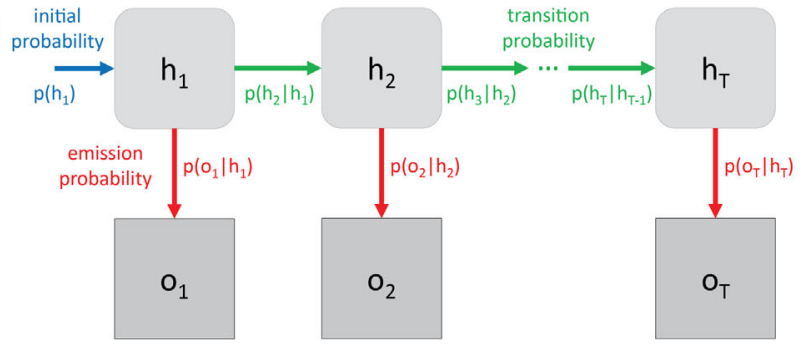

(b)

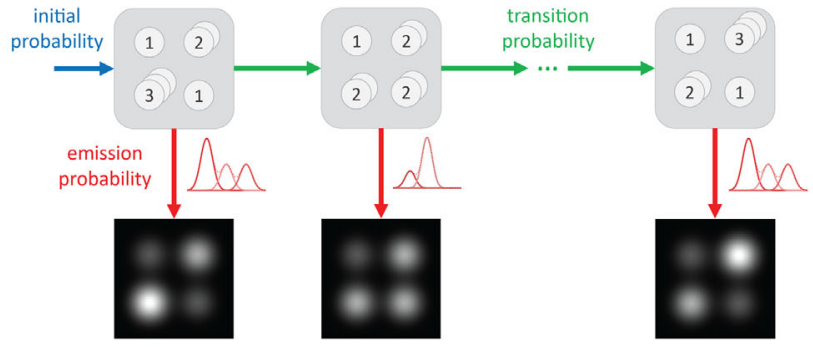

FIG. 1. (a) Graphical representation of a hidden Markov model, which consists of a hidden state sequence (top row) and an observed sequence (bottom row), connected through the emission probability (red). The hidden states can have different possible values over time according to initial (blue) and transition probabilities (green). (b) The hidden Markov model for atom counting models the number of atoms in each atomic column of the nanoparticle as the hidden states (top row) and the scattering cross sections obtained from the ADF STEM images as the observations (bottom row). 
knowledge from image simulations is incorporated by imposing a linear scaling between the average scattering cross sections and the scattering cross section resulting from image simulations:

$$
\mu_{g}=a \mathcal{M}_{g}
$$

In this expression, $\mathcal{M}_{g}$ is the scattering cross section resulting from image simulations and $a$ is a linear scaling parameter that allows us to compensate for a possible mismatch between experiment and simulation, such as intensity changes due to a slightly different detector inner angle or the intensity loss caused by small sample tilt $[46,57]$. This approach was introduced for single frames as the hybrid method for atom counting, yielding improved atom-counting reliability, especially at low electron doses, compared to alternative approaches [46].

In order to retrieve the hidden state sequence from the observed sequence, the parameters of the factorial hidden Markov model for atom counting are estimated. The unknown parameters are

$$
\boldsymbol{\Omega}=\left(l_{0}, \ldots, l_{G-1}, A_{00}, \ldots, A_{G, G-1}, a, \sigma\right) .
$$

Since $\sum_{g=0}^{G} l_{g}=1$ and $\sum_{g=0}^{G} A_{j g}=1 \forall 0 \leq j \leq G, G+2$ parameters $\left(l_{G}\right.$ and $A_{j G}$ with $\left.0 \leq j \leq G\right)$ could be omitted from the parameter vector $\boldsymbol{\Omega}$. The parameters $\boldsymbol{\Omega}$ can be estimated using the maximum likelihood estimator [58]. Therefore, the complete data likelihood function of the unknown parameter vector for the observed and hidden data, which follows from Eq. (1), has to be maximized:

$$
L(\boldsymbol{\Omega} ; \boldsymbol{H}, \boldsymbol{O})=p\left(\boldsymbol{h}_{1}\right) \prod_{t=2}^{T} p\left(\boldsymbol{h}_{t} \mid \boldsymbol{h}_{t-1}\right) \prod_{t^{\prime}=1}^{T} p\left(\boldsymbol{o}_{t^{\prime}} \mid \boldsymbol{h}_{t^{\prime}}\right) .
$$

The likelihood can be maximized analytically, by solving the likelihood equation

$$
\frac{\partial \log L(\boldsymbol{\Omega} ; \boldsymbol{H}, \boldsymbol{O})}{\partial \boldsymbol{\Omega}}=0 .
$$

This equation is solved iteratively, using an expectationmaximization algorithm, usually called the Baum-Welch algorithm in the context of hidden Markov models [50]. Using the estimated parameters, the hidden state sequence with the joint highest probability is retrieved using a path backtracking algorithm, called the Viterbi algorithm $[59,60]$. More details about both algorithms are provided in Sec. I of the Supplemental Material [55].

In order to illustrate the benefits of this newly developed hidden Markov model for atom counting from a time series, we compare its performance to the hybrid method [46]. For the hybrid method, the scattering cross sections of all frames of the time series are jointly analyzed, such that the counting results are based on the same set of observations as the hidden Markov model. The counting results are then extracted per frame from this so-called collective analysis $[61,62]$. We simulated scattering cross sections corresponding to hypothetic ADF STEM time series with 40 frames of a changing Pt nanoparticle with 215 atomic columns, and a thickness up to 15 atoms, similar to the experimental example that will be discussed further in this Letter. The number of atoms in a column can change by \pm 1 from frame to frame throughout the time series, with a probability of $10 \%$. An example of the 3D atomic structure of the $\mathrm{Pt}$ nanoparticle and how it changes over time is shown in Fig. 2(a). More details about the simulation can be found in Sec. II of the Supplemental Material [55]. In Fig. 2(b), the average percentage of correctly counted atomic columns by both methods, with a $95 \%$ confidence interval, is evaluated as a function of the electron dose. The hidden Markov (a)

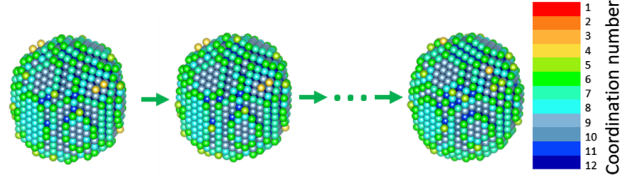

(b)

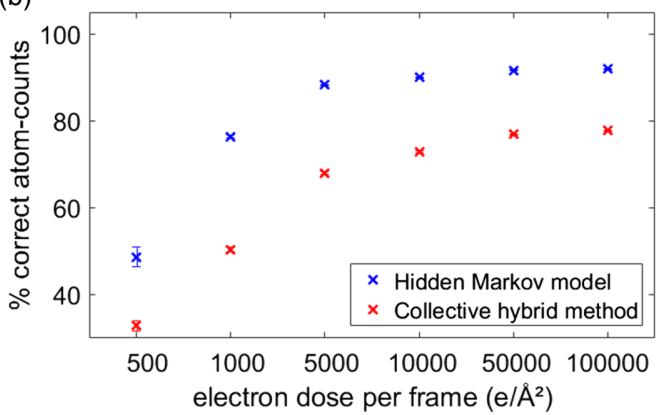

Ground truth
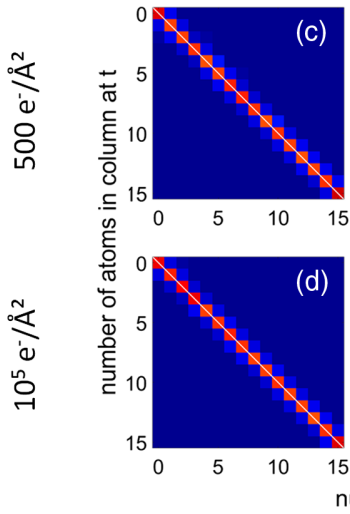

Hidden Markov model
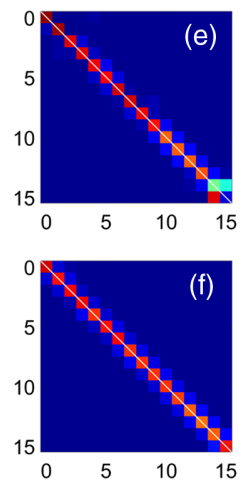

(f)

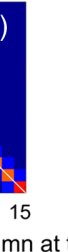

Collective hybrid method
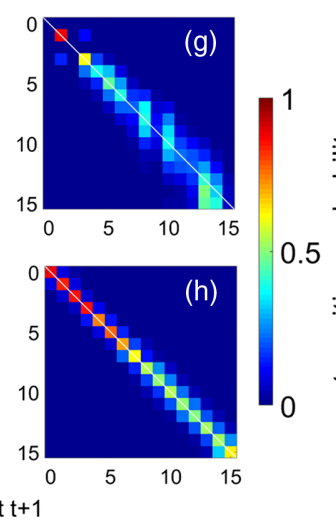

FIG. 2. (a) Example of the 3D atomic structure of the changing Pt nanoparticle. (b) Percentage of correctly counted atomic columns, with a 95\% confidence interval as a function of the electron dose in each individual frame. (c)-(h) Ground truth and estimated transition matrices using the hidden Markov model analysis and the collective hybrid method with electron dose $5 \times 10^{2}$ and $10^{5} e^{-} / \AA^{2}$. 
model counts the number of atoms in each column more accurately, both at low electron doses, where Poisson noise dominates, and at high electron dose, where the scan distortion is the dominant noise contribution [63]. The improved performance of the hidden Markov model is a direct consequence of using the Viterbi algorithm to retrieve the counting results. The most likely state sequence obtained by this algorithm employs the extra information from the transition probabilities to unravel the states and is therefore not limited strictly by the overlap of the Gaussian distributions around the average scattering cross sections of columns with different thicknesses.

In Figs. 2(c) and 2(d), the underlying transition matrices $\boldsymbol{A}$ are shown for a noise realization at low and high electron doses, respectively. In Figs. 2(e)-2(h), we show the estimated transition matrices using both methods for the respective low and high dose noise realizations. The transition matrix for the collective hybrid method was estimated by considering the obtained atom-counting results as a Markov chain. The transition matrix summarizes the estimated structural changes of the nanoparticle: diagonal elements correspond to the probabilities that the number of atoms in an atomic column with a given thickness does not change from one frame to the next and off-diagonal elements in the lower and upper triangle correspond to the probabilities for an atomic column to lose or gain atoms respectively. From the comparison of Figs. 2(g) and 2(h) with the respective ground truth in Figs. 2(c) and 2(d), it is clear that the collective hybrid method overinterprets intensity variations during the time series as actual thickness changes, both at low and high electron doses. From Fig. 2(e) and 2(f) on the other hand, it is clear that the hidden Markov model far more accurately retrieves the transition probabilities, and therefore opens up possibilities for a reliable quantification of dynamic structural changes of nanoparticles at the atomic scale.

Next, we apply this to an experimental time series of a catalyst Pt nanoparticle. ADF STEM images were recorded on a JEOL ARM200CF fitted with a probe-aberration corrector using an acceleration voltage of $200 \mathrm{kV}$, a probe convergence angle of $22.48 \mathrm{mrad}$, an annular detector ranging from 52-248 mrad, a dwell time of $4 \mu \mathrm{s}$ and an electron dose of $1.38 \times 10^{4} e^{-} / \AA^{2}$ per frame. All images of the time series are shown in Fig. S1 of the Supplemental Material [55]. The images from the time series were corrected for drift and other distortions using nonrigid registration [64]. Coordinates of the atomic columns were selected in each frame using the maximum a posteriori probability (MAP) rule for atomic column detection introduced in [65]. As such, we could reliably determine all atomic columns present in the nanoparticle throughout the time series. During the time series, the Pt nanoparticle tilts slightly away from zone axis orientation and back, which affects the scattering cross sections [57]. However, the hidden Markov model only estimates one linear scaling (a)
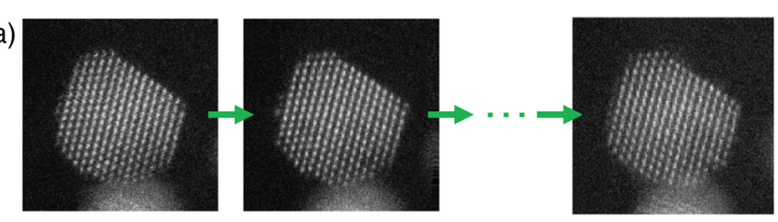

(b)

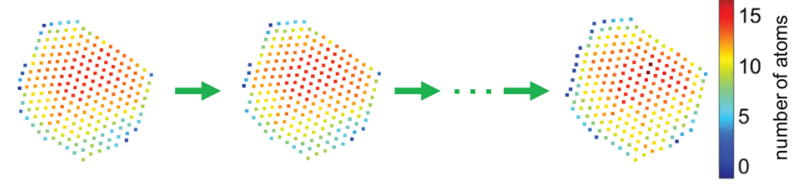

FIG. 3. (a) The experimental ADF STEM time series of a Pt nanoparticle. (b) From the estimated hidden Markov model, the hidden state sequence is retrieved.

parameter for all frames of the time series. Therefore, the scattering cross sections of the individual frames need to be compensated for tilt, prior to the hidden Markov model analysis. This is done by using a linear scaling of the scattering cross sections of the individual frames [46], assuming that the total number of atoms in the nanoparticle remains constant throughout the time series. This assumption is valid since the threshold energy for sputtering $\mathrm{Pt}$ atoms from a convex surface with step sites is $379 \mathrm{keV}$ [16], well above the incident electron energy of $200 \mathrm{keV}$. We therefore do not expect sputtering of atoms from the surface, only surface diffusion [63]. Next, dynamic structural changes are determined from the time series analysis using a hidden Markov model, of which the results are shown schematically in Fig. 3. The counting results for all frames are shown in Fig. S2 of the Supplemental Material [55].

The HAADF STEM projection images reveal the faceted shape of the Pt nanoparticle. The $\{111\}$ facets, on occasion decorated by additional atoms [66], are indicated by green crosses in Fig. 4(a). Using the counting results from our hidden Markov model analysis, we can now quantify the evolution of the number of atoms in these facets for each frame of the time series [Fig. 4(b)]. While the total number of atoms in the Pt nanoparticle remains the same, the number of atoms in the $\{111\}$ facets along the beam direction decreases and the Pt nanoparticle gradually loses its faceted morphology during electron beam irradiation.
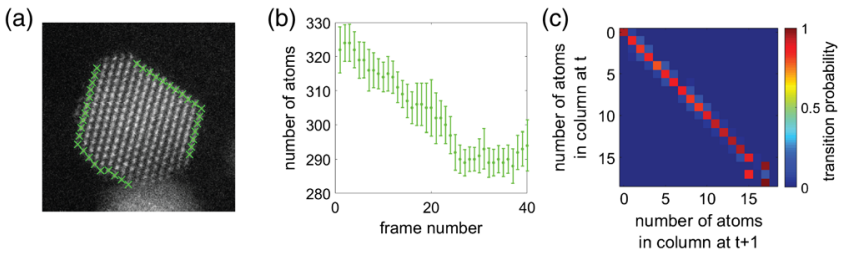

FIG. 4. (a) The Pt nanoparticle shows clear facets along the beam direction. Atomic columns in $\{111\}$ facets are indicated. (b) Number of atoms in the $\{111\}$ facets indicated in (a). (c) Estimated transition probability matrix. 
This result is consistent with earlier observations of the same type of Pt nanoparticles [44] and can be explained by beam-induced surface diffusion. The hidden Markov model analysis has the added advantage that it enables us to quantify the probability for surface diffusion. From the transition probabilities shown in Fig. 4(c) it follows that the average probability for a surface atom to move to another column equals $4.6 \%$. Taking into account the electron dose $[63,67]$, an experimental value for the average cross section for surface diffusion $\hat{\sigma}=3.3 \times 10^{-6} \AA^{2}$, can even be determined. This cross section for surface diffusion includes the contributions of different migration mechanisms, such as hopping, atomic exchange, and vacancy diffusion, and from different types of surfaces $[68,69]$. This value is of great importance in order to unravel dominant mechanisms and surfaces in the diffusion process and to gain new insights in surface related phenomena such as catalysis and nanoparticle growth.

In conclusion, we present a new statistical framework to reliably count the number of atoms in the atomic columns of a pure-element nanostructure in each frame of an ADF STEM time series using the so-called factorial hidden Markov model. As a proof of concept, we show that the performance of this new method significantly surpasses that of the current method for atom counting. This improved performance could be achieved since the hidden Markov model explicitly models the dynamics of the system. The new method is applied to an experimental time series of ADF STEM images of a catalyst Pt nanoparticle, and reveals the loss of the Pt nanoparticle's faceted morphology during the time series, due to the electron beam irradiation. Furthermore, our novel analysis approach enables us to quantify the probability and cross section for surface diffusion from a time series of experimental ADF STEM images. The hidden Markov model for atom counting therefore holds promise for a reliable quantification of dynamic structural changes by adatom dynamics, surface diffusion, beam effects, or during in situ experiments. The hidden Markov model was implemented in the freely available StatSTEM software [2].

This project has received funding from the European Research Council (ERC) under the European Union's Horizon 2020 research and innovation programme (Grant Agreement No. 770887 and No. 823717 ESTEEM3). The authors acknowledge financial support from the Research Foundation Flanders (FWO, Belgium) through grants to A. D. w. and A. D. B. and Projects No. G.0502.18N and No. EOS 30489208. L. J. acknowledges the SFI AMBER Centre for support. A. V. and P. D. N. acknowledge the UK Engineering and Physical Sciences Council (EPSRC) for support (EP/K040375/1 and 1772738). A. V. also acknowledges Johnson-Matthey for support. We would like to thank Brian Theobald and Jonathan Sharman from JMTC Sonning for provision of the Pt sample. *sandra.vanaert@uantwerpen.be

†Present address: Johnson Matthey Technology Centre, Blounts Court Road, Sonning Common, RG4 9NH Reading, United Kingdom.

[1] P. L. Galindo, S. Kret, A. M. Sanchez, J.-Y. Laval, A. Yáñez, J. Pizarro, E. Guerrero, T. Ben, and S. I. Molina, The Peak Pairs algorithm for strain mapping from HRTEM images, Ultramicroscopy 107, 1186 (2007).

[2] A. De Backer, K. H. W. van den Bos, W. Van den Broek, J. Sijbers, and S. Van Aert, StatSTEM: An efficient approach for accurate and precise model-based quantication of atomic resolution electron microscopy images, Ultramicroscopy 171, 104 (2016).

[3] Y. Wang, U. Salzberger, W. Sigle, E. Suyolcu, and P. van Aken, Oxygen octahedra picker: A software tool to extract quantitative information from STEM images, Ultramicroscopy 168, 46 (2016).

[4] M. Nord, P. E. Vullum, I. MacLaren, T. Tybell, and R. Holmestad, Atomap: A new software tool for automated analysis of atomic resolution images using two-dimensional Gaussian fitting, Adv. Struct. Chem. Imaging 3, 9 (2017).

[5] J. Madsen, P. Liu, J. Kling, J. B. Wagner, T. W. Hansen, O. Winther, and J. Schiøtz, A deep learning approach to identify local structures in atomic-resolution transmission electron microscopy images, Adv. Theory Simul. 1, 1800037 (2018).

[6] F. de la Peña et al., hyperspy/hyperspy: Hyperspy v1.5.2, 2019.

[7] H. Chang, M. Saito, T. Nagai, Y. Liang, Y. Kawazoe, Z. Wang, H. Wu, K. Kimoto, and Y. Ikuhara, Single adatom dynamics at monatomic steps of free-standing few-layer reduced graphene, Sci. Rep. 4, 6037 (2015).

[8] T. Furnival, D. Knez, E. Schmidt, R. K. Leary, G. Kothleitner, F. Hofer, P. D. Bristowe, and P. A. Midgley, Adatom dynamics and the surface reconstruction of $\mathrm{Si}(110)$ revealed using time-resolved electron microscopy, Appl. Phys. Lett. 113, 183104 (2018).

[9] A. Surrey, D. Pohl, L. Schultz, and B. Rellinghaus, Quantitative measurement of the surface self-diffusion on Au nanoparticles by aberration-corrected transmission electron microscopy, Nano Lett. 12, 6071 (2012).

[10] S. Schneider, A. Surrey, D. Pohl, L. Schultz, and B. Rellinghaus, Atomic surface diffusion on Pt nanoparticles quantified by high-resolution transmission electron microscopy, Micron 63, 52 (2014).

[11] V. Cherepanov and B. Voigtländer, Influence of material, surface reconstruction, and strain on diffusion at the Ge(111) surface, Phys. Rev. B 69, 125331 (2004).

[12] P. A. Buffat, Electron diffraction and HRTEM studies of multiply-twinned structures and dynamical events in metal nanoparticles: facts and artefacts, Mater. Chem. Phys. 81, 368 (2003).

[13] J. Yu, X. Li, J. Miao, W. Yuan, S. Zhou, B. Zhu, Y. Gao, H. Yang, Z. Zhang, and Y. Wang, Atomic mechanism in layerby-layer growth via surface reconstruction, Nano Lett. 19, 4205 (2019).

[14] S. Iijima and T. Ichihashi, Structural Instability of Ultrafine Particles of Metals, Phys. Rev. Lett. 56, 616 (1986). 
[15] P. E. Batson, Motion of gold atoms on carbon in the aberration-corrected STEM, Microsc. Microanal. 14, 89 (2008).

[16] R. F. Egerton, R. McLeod, F. Wang, and M. Malac, Basic questions related to electron-induced sputtering in the TEM, Ultramicroscopy 110, 991 (2010).

[17] J. Lee, W. Zhou, J. Pennycook, J.-C. Idrobo, and S. T. Pantelides, Direct visualization of reversible dynamics in a $\mathrm{Si}_{6}$ cluster embedded in a graphene pore, Nat. Commun. $\mathbf{4}$, 1650 (2013).

[18] M. L. Taheri, E. A. Stach, I. Arslan, P. A. Crozier, B. C. Kabius, T. LaGrange, A. M. Minor, S. Takeda, M. Tanase, J. B. Wagner, and R. Sharma, Current status and future directions for in situ transmission electron microscopy, Ultramicroscopy 170, 86 (2016).

[19] T. Altantzis, I. Lobato, A. De Backer, A. Béché, Y. Zhang, S. Basak, M. Porcu, Q. Xu, A. Sánchez-Iglesias, L. M. LizMarzán, G. Van Tendeloo, S. Van Aert, and S. Bals, Threedimensional quantification of the facet evolution of $\mathrm{Pt}$ nanoparticles in a variable gaseous environment, Nano Lett. 19, 477 (2019).

[20] M. R. Armstrong, K. Boyden, N. D. Browning, G. H. Campbell, J. D. Colvin, W. J. DeHope, A. M. Frank, D. J. Gibson, F. Hartemann, J.S. Kim, W. E. King, T. B. LaGrange, B. J. Pyke, B. W. Reed, R. M. Shuttlesworth, B. C. Stuart, and B.R. Torralva, Practical considerations for high spatial and temporal resolution dynamic transmission electron microscopy, Ultramicroscopy 107, 356 (2007).

[21] N. D. Browning, M. A. Bonds, G. H. Campbell, J. E. Evans, T. LaGrange, K. L. Jungjohann, D. J. Masiel, J. McKeown, S. Mehraeen, B. W. Reed, and M. Santala, Recent developments in dynamic transmission electron microscopy, Curr. Opin. Solid State Mater. Sci. 16, 23 (2012).

[22] B. Barwick, H. S. Park, O.-H. Kwon, and A. H. Baskin, J. S. Zewail, 4D imaging of transient structures and morphologies in ultrafast electron microscopy, Science 322, 1227 (2008).

[23] B. J. Siwick, J. R. Dwyer, R. E. Jordan, and R. J. D. Miller, An atomic-level view of melting using femtosecond electron diffraction, Science 302, 1382 (2003).

[24] A. H. Zewail, 4D ultrafast electron diffraction, crystallography, and microscopy, Annu. Rev. Phys. Chem. 57, 65 (2006).

[25] C.-Y. Ruan, Y. Murooka, R. K. Raman, and R. A. Murdick, Dynamics of size-selected gold nanoparticles studied by ultrafast electron nanocrystallography, Nano Lett. 7, 1290 (2007).

[26] C.-Y. Ruan, Y. Murooka, R. K. Raman, and R. A. Murdick, The development and applications of ultrafast electron nanocrystallography, Microsc. Microanal. 15, 323 (2009).

[27] A. A. Ischenko and S. A. Aseyev, Ultrafast electron crystallography and nanocrystallography, in Time-Resolved Electron Diffraction-For Chemistry, Biology and Materials Science (Elsevier, 2014).

[28] C. Rischel, A. Rousse, I. Uschmann, P.-A. Albouy, J.-P. Geindre, P. Audebert, J.-C. Gauthier, E. Förster, J.-L. Martin, and A. Antonetti, Femtosecond time-resolved $\mathrm{X}$-ray diffreaction from laser-heated organic films, Nature (London) 390, 490 (1997).
[29] A. M. Lindenberg, S. L. Johnson, and D. A. Reis, Visualization of atomic-scale motions in materials via femtosecond X-ray scattering techniques, Annu. Rev. Mater. Res. 47, 425 (2017).

[30] C. L. Jia, S. B. Mi, J. Barthel, D. W. Wang, R. E. Dunin-Borkowski, K. W. Urban, and A. Thust, Determination of the 3D shape of a nanoscale crystal with atomic resolution from a single image, Nat. Mater. 13, 1044 (2014).

[31] M. Linck, B. Freitag, S. Kujawa, M. Lehmann, and T. Niermann, State of the art in atomic resolution off-axis electron holography, Ultramicroscopy 116, 13 (2012).

[32] S. Van Aert, K. J. Batenburg, M. D. Rossell, R. Erni, and G. Van Tendeloo, Three-dimensional atomic imaging of crystalline nanoparticles, Nature (London) 470, 374 (2011).

[33] S. Van Aert, A. De Backer, G. T. Martinez, B. Goris, S. Bals, G. Van Tendeloo, and A. Rosenauer, Procedure to count atoms with trustworthy single-atom sensitivity, Phys. Rev. B 87, 064107 (2013).

[34] M. Isaacson, D. Kopf, M. Utlaut, N. W. Parker, and A. V. Crewe, Direct observations of atomic diffusion by scanning transmission electron microscopy, Proc. Natl. Acad. Sci. U.S.A. 74, 1802 (1977).

[35] H.-G. Liao, D. Zherebetskyy, H. Xin, C. Czarnik, P. Ercius, H. Elmlund, M. Pan, L.-W. Wang, and H. Zheng, Facet development during platinum nanocube growth, Science 345, 916 (2014).

[36] J. Kotakoski, C. Mangler, and J. C. Meyer, Imaging atomiclevel random walk of a point defect in graphene, Nat. Commun. 5, 3991 (2014).

[37] R. Mishra, R. Ishikawa, A. R. Lupini, and S. J. Pennycook, Single-atom dynamics in scanning transmission electron microscopy, MRS Bull. 42, 644 (2017).

[38] P. Liu, T. Wu, J. Madsen, J. Schiøtz, J. B. Wagner, and T. W. Hansen, Transformations fo supported gold nanoparticles observed by in situ electron microscopy, Nanoscale 11, 11885 (2019).

[39] J. Zhou, Y. Yang, Y. Yang, D. S. Kim, A. Yuan, X. Tian, C. Ophus, F. Sun, A. K. Schmid, M. Nathanson, H. Heinz, Q. An, H. Zeng, P. Ercius, and J. Miao, Observing crystal nucleation in four dimensions using atomic electron tomography, Nature (London) 570, 500 (2019).

[40] S. Van Aert, J. Verbeeck, R. Erni, S. Bals, M. Luysberg, D. Van Dyck, and G. Van Tendeloo, Quantitative atomic resolution mapping using high-angle annular dark field scanning transmission electron microscopy, Ultramicroscopy 109, 1236 (2009).

[41] H. E, K. E. MacArthur, T. J. Pennycook, E. Okunishi, A. J. D'Alfonso, N. R. Lugg, L. J. Allen, and P. D. Nellist, Probe integrated scattering cross sections in the analysis of atomic resolution HAADF STEM images, Ultramicroscopy 133, 109 (2013).

[42] A. De Backer, A. De wael, J. Gonnissen, and S. Van Aert, Optimal experimental design for nano-particle atom-counting from high-resolution STEM images, Ultramicroscopy 151, 46 (2015).

[43] J. M. LeBeau, S. D. Findlay, L. J. Allen, and S. Stemmer, Standardless atom counting in scanning transmission electron microscopy, Nano Lett. 10, 4405 (2010).

[44] L. Jones, K. E. MacArthur, V. T. Fauske, A. T. J. van Helvoort, and P. D. Nellist, Rapid estimation of catalyst nanoparticle 
morphology and atomic-coordination by high-resolution Z-contrast electron microscopy, Nano Lett. 14, 6336 (2014).

[45] A. De Backer, G. T. Martinez, A. Rosenauer, and S. Van Aert, Atom counting in HAADF STEM using a statistical model-based approach: Methodology, possibilities, and inherent limitations, Ultramicroscopy 134, 23 (2013).

[46] A. De wael, A. De Backer, L. Jones, P. D. Nellist, and S. Van Aert, Hybrid statistics-simulations based method for atom-counting using scanning transmission electron microscopy, Ultramicroscopy 177, 69 (2017).

[47] S. Bals, S. Van Aert, C. P. Romero, K. Lauwaet, M. J. Van Bael, B. Schoeters, B. Partoens, E. Yücelen, P. Lievens, and G. Van Tendeloo, Atomic scale dynamics of ultrasmall germanium clusters, Nat. Commun. 3, 897 (2012).

[48] J. J. Geuchies, C. van Overbeek, W. H. Evers, B. Goris, A. De Backer, G. P. Gantapara, F. T. Rabouw, J. Hilhorst, J. L. Peters, O. Konovalov, A. V. Petukhov, M. Dijkstra, L. D. A. Siebbeles, S. Van Aert, S. Bals, and D. Vanmaekelbergh, In situ study of consecutive phase transitions in the formation of atomatomic coherent two-dimensional supperlattices from nanocrystals, Nat. Mater. 15, 1248 (2016).

[49] A. De Backer, L. Jones, I. Lobato, T. Altantzis, B. Goris, P. D. Nellist, S. Bals, and S. Van Aert, Three-dimensional atomic models from a single projection using Z-contrast imaging: verification by electron tomography and opportunities, Nanoscale 9, 8791 (2017).

[50] L. R. Rabiner, A tutorial on hidden Markov models and selected applications in speech recognition, Proc. IEEE 77, 257 (1989).

[51] J. A. Bilmes, A Gentle Tutorial of the EM Algorithm and its Application to Parameter Estimation for Gaussian Mixture and Hidden Markov Models, International Computer Science Institute Berkeley, Report No. TR-97-021, 1998.

[52] S. R. Eddy, What is a hidden Markov model?, Nat. Biotechnol. 22, 1315 (2004).

[53] C. Bishop, Pattern Recognition and Machine Learning (Springer-Verlag, New York, 2006).

[54] P. Dymarski, Hidden Markov Models, Theory and Applications (InTech, Rijeka, Croatia, 2011).

[55] See Supplemental Material at http://link.aps.org/ supplemental/10.1103/PhysRevLett.124.106105 for technical details on algorithms, simulation and experimental setup, and all images of the experimental time series of the Pt nanoparticle.

[56] Z. Ghahramani and M. I. Jordan, Factorial hidden Markov models, Mach. Learn. 29, 245 (1997).
[57] K. E. MacArthur, A. J. D’Alfonso, D. Ozkaya, L. Allen, and P. Nellist, Optimal ADF STEM imaging parameters for tiltrobust image quantification, Ultramicroscopy 156, 1 (2015).

[58] G. McLachlan and D. Peel, in Finite Mixture Models, Wiley Series in Probability and Statistics (John Wiley and Sons, Inc., New York, 2000).

[59] A. J. Viterbi, Error bounds for convolutional codes and an asymptotically optimum decoding algorithm, IEEE Trans. Inf. Theory 13, 260 (1967).

[60] G. D. Forney, The Viterbi algorithm, IEEE Trans. Inf. Theory 61, 268 (1973).

[61] A. De Backer, G. T. Martinez, K. E. MacArthur, L. Jones, A. Béché, P. D. Nellist, and S. Van Aert, Dose limited reliability of quantitative annular dark field scanning transmission electron microscopy for nano-particle atomcounting, Ultramicroscopy 151, 56 (2015).

[62] A. M. Varambhia, L. Jones, A. De Backer, V. T. Fauske, S. Van Aert, D. Ozkaya, and P. D. Nellist, Quantifying a heterogeneous $\mathrm{Ru}$ catalyst on carbon black using $\mathrm{ADF}$ STEM, Part. Part. Syst. Charact. 33, 438 (2016).

[63] S. Van Aert, A. De Backer, L. Jones, G. T. Martinez, A. Béché, and P. D. Nellist, Control of Knock-On Damage for 3D Atomic Scale Quantication of Nanostructures: Making Every Electron Count in Scanning Transmission Electron Microscopy, Phys. Rev. Lett. 122, 066101 (2019).

[64] L. Jones, H. Yang, T. J. Pennycook, M. S. J. Marshall, S. Van Aert, N. D. Browning, M. R. Castell, and P. D. Nellist, Smart Align - a new tool for robust non-rigid registration of scanning microscope data, Adv. Struct. Chem. Imaging 1, 8 (2015).

[65] J. Fatermans, A. J. den Dekker, K. Müller-Caspary, I. Lobato, C. M. O'Leary, P. D. Nellist, and S. Van Aert, Single Atom Detection from Low Contrast-to-Noise Ratio Electron Microscopy Images, Phys. Rev. Lett. 121, 056101 (2018).

[66] Z. W. Wang and R.E. Palmer, Mass spectrometry and dynamics of gold adatoms observed on the surface of size-selected Au nanoclusters, Nano Lett. 12, 91 (2012).

[67] R. F. Egerton, Beam-induced motion of Adatoms in the transmission electron microscope, Microsc. Microanal. 19, 479 (2013).

[68] K. Oura, M. Katayama, A. V. Zotov, V. G. Lifshits, and A. A. Saranin, Elementary processes at surfaces II. Surface diffusion, in Surface Science-An Introduction (Springer, Berlin, Heidelberg, 2003).

[69] R. F. Egerton, Radiation damage to organic and inorganic specimens in the TEM, Micron 119, 72 (2019). 